\title{
Um Novo Algoritmo de Balanceamento Espectral Entre Grupos de Núcleos para Redes Ópticas Elásticas com Multiplexação por Divisão Espacial
}

\author{
Paulo E. R. Araujo ${ }^{1}$, Jurandir C. Lacerda Jr. ${ }^{1,2}$, André C. B. Soares ${ }^{1}$ \\ ${ }^{1}$ Departamento de Computação - Universidade Federal do Piauí (UFPI) \\ Teresina - PI \\ ${ }^{2}$ Instituto Federal de Educação, Ciência e Tecnologia do Piauı (IFPI) \\ Corrente - PI \\ pauloaraujo@ufpi.edu.br
}

\begin{abstract}
The adoption of spatial division multiplexing in elastic optical networks has created new challenges for the allocation of optical resources. The inter-core crosstalk appears when the same optical frequency is used in adjacent cores. In this paper, the inter-core spectral distancing algorithm (ADEIN) is proposed. To avoid crosstalk, ADEIN balances the use of resources in order to prioritize certain groups of cores and spectral slices. The proposal achieved a minimal improvement in the bandwidth blocking probability of $47.01 \% \mathrm{com}$ pared to other algorithms in the literature. For the circuit blocking probability, the improvement was at least $44.64 \%$.
\end{abstract}

Resumo. A introdução da multiplexação por divisão espacial nas redes ópticas elásticas proporcionou o surgimento de novos desafios para a alocação dos recursos ópticos. $O$ crosstalk inter-núcleo surge quando uma mesma frequência óptica é utilizada em núcleos adjacentes. Neste artigo é proposto o algoritmo de distanciamento espectral inter-núcleos (ADEIN). Com o objetivo de evitar o crosstalk, o ADEIN realiza um balanceamento no uso dos recursos de modo a priorizar determinados grupos de núcleos e faixas espectrais. A proposta obteve uma melhora mínima na probabilidade de bloqueio de banda de 47,01\% em relação a outros algoritmos da literatura. Para a probabilidade de bloqueio de circuito, a melhora foi de pelo menos 44,64\%.

\section{Introdução}

Nos últimos anos, a quantidade de usuários da rede de internet tem crescido de maneira contínua por todo o globo [Savva et al. 2018]. Esse massivo crescimento reverbera em um aumento constante no fluxo de dados trafegados. Com a popularização dos aplicativos de streaming, do trabalho remoto, da computação em nuvem e entre outros, faz-se necessário uma infraestrutura de rede que comporte a necessidade de todos os usuários. Afim de suprir toda essa demanda, as fibras ópticas são uma ótima alternativa para compor os backbones das redes de transporte da internet [Santos et al. 2019].

As redes ópticas elásticas (Elastic Optical Network - EON) [Jinno et al. 2009] são uma tecnologia de transmissão promissora para atender ao crescente fluxo de dados 
trafegado. Nesse tipo de rede, o espectro óptico é divido em pequenas porções denominadas slots de frequência, que podem ser combinados para formar circuitos ópticos que atendam diferentes larguras de banda. Esse tipo de abordagem permite um uso mais eficiente do espectro óptico quando comparado com o modelo de grade fixa das redes ópticas com multiplexação por comprimento de onda (Wavelength Division Multiplexing - WDM) [Chatterjee et al. 2015].

Mais recentemente, um novo modelo de multiplexação nomeado multiplexação por divisão espacial (Space Division Multiplexing - SDM) [Klinkowski and Zalewski 2019, Oliveira and Fonseca 2019] tem se mostrado mais eficiente na disponibilização de recursos para a rede. Nesse modelo de multiplexação, a implementação da dimensão do espaço é feita com a adição de diversos núcleos dentro de uma única fibra. A Figura 1 apresenta a diferença dos modelos de multiplexação, como pode-se observar, os múltiplos núcleos aumentam a capacidade da rede suportar volumes maiores de tráfego.

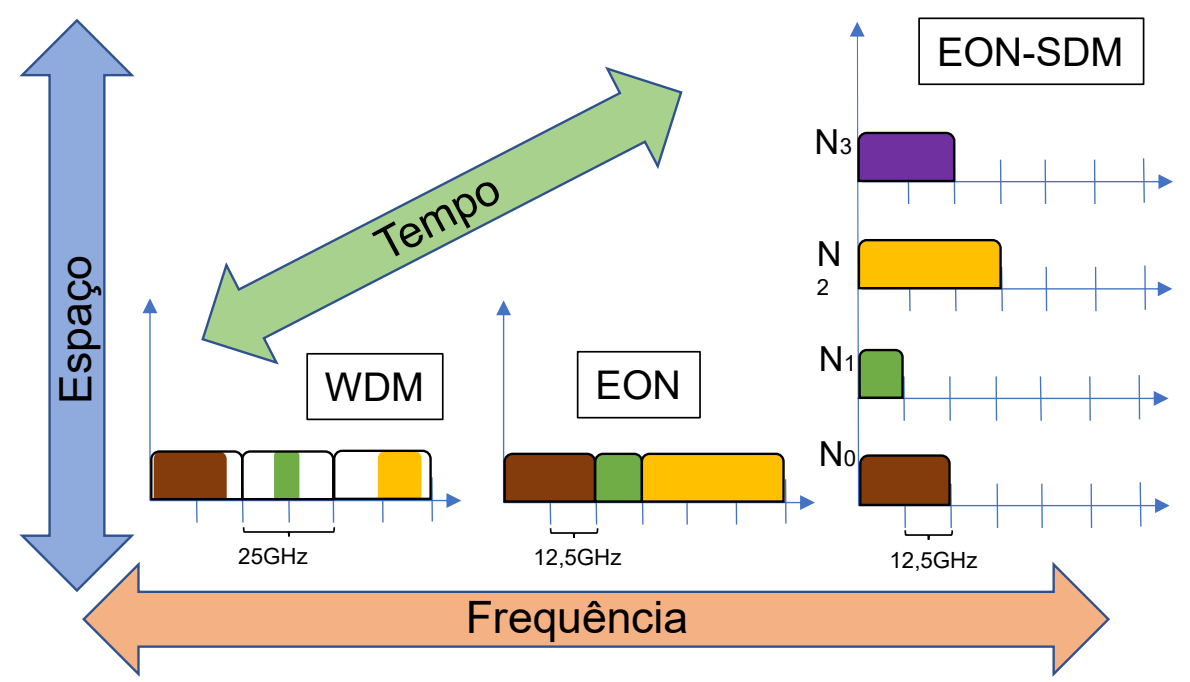

Figura 1. Três Diferentes Modelos de Provisionamento de Recurso.

Apesar de proporcionar mais recursos disponíveis para alocação, as redes EONSDM trazem consigo problemas na camada física. Um deles é o crosstalk (XT), que ocorre quando slots de mesma frequência são alocados em núcleos adjacentes. Assim, requisições podem ser bloqueadas por gerarem um nível de XT inaceitável na rede. O bloqueio por crosstalk ocorre quando, no processo de atendimento de uma nova requisição, caso o circuito fosse estabelecido, a rede passaria a ter níveis de XT que ultrapasse os limiares mínimos de qualidade. De maneira geral, o plano de controle da rede deve gerenciar todo esse processo, buscando impedir o estabelecimento de novos circuitos que resultem em níveis de XT inaceitáveis.

Para o estabelecimento de um circuito óptico em uma rede EON-SDM devese resolver o problema de Roteamento, Formato de Modulação e Alocação de Núcleo e Espectro (Routing, Modulation Format, Core and Spectrum Allocation - RMCSA [Trindade and da Fonseca 2020]. O problema RMCSA consiste em: i) Escolher uma rota entre o nó origem e o nó destino; ii) Definir um formato de modulação para envio dos 
bits de sinais; iii) Selecionar um núcleo e uma faixa de slots para alocação do circuito, também conhecido como subproblema CSA(Core and Spectrum Allocation).

Este trabalho foca no subproblema CSA em um cenário de tráfego dinâmico de uma rede EON-SDM. A principal contribuição deste artigo é a proposta de um algoritmo para a redução dos níveis de crosstalk na rede utilizando: i) um método de escolha de núcleo por pontuação e ii) subdivisão do espectro óptico em diferentes regiões prioritárias. O Algoritmo proposto é chamado de Algoritmo de Distanciamento Espectral Inter-Núcleos (ADEIN). O algoritmo obteve menor probabilidade de bloqueio de circuito e de banda devido a diminuição da ocorrência de crosstalk. Nos estudos de simulação realizados neste artigo, o ADIEN apresentou melhor desempenho quando comparado com outros trabalhos da literatura. Para medir o desempenho do algoritmo, foram utilizada as métricas de probabilidade de bloqueio de banda, probabilidade de bloqueio de circuito e probabilidade de bloqueio de banda por crosstalk.

O restante deste artigo está organizado da seguinte forma. A Seção 2 apresenta conceitos das redes ópticas elásticas com multiplexação por divisão espacial e suas características. A Seção 3 apresenta um levantamento sobre o estado da arte da literatura em relação à redes EON-SDM. Na Seção 4 o algoritmo proposto é descrito. A Seção 5 apresenta a avaliação do desempenho do algoritmo proposto. Por fim, na Seção 6 são apresentadas as conclusões e trabalhos futuros.

\section{Redes Ópticas Elásticas Com Multiplexação por Divisão Espacial}

Em uma rede com multiplexação por divisão espacial, a comunicação entre pares de nós origem e destino é feita utilizando um dos diversos núcleos dispostos em uma única fibra, também conhecida como Multi-Core Fiber - MCF. Cada núcleo contem uma faixa de espectro óptico que é dividida em pequenos grupos de $12,5 \mathrm{GHz}$ cada, denominadas slots de frequência. [Fujii et al. 2014]

Para este trabalho, será considerada uma MCF contendo sete núcleos. Esse modelo de fibra é composto por um núcleo central e seis núcleos de borda com uma distância $\Lambda$ entre cada núcleo. Este tipo de abordagem para fibras MCF tem sido frequentemente explorada na literatura [Oliveira and Fonseca 2018] [Ehsani Moghaddam et al. 2019].

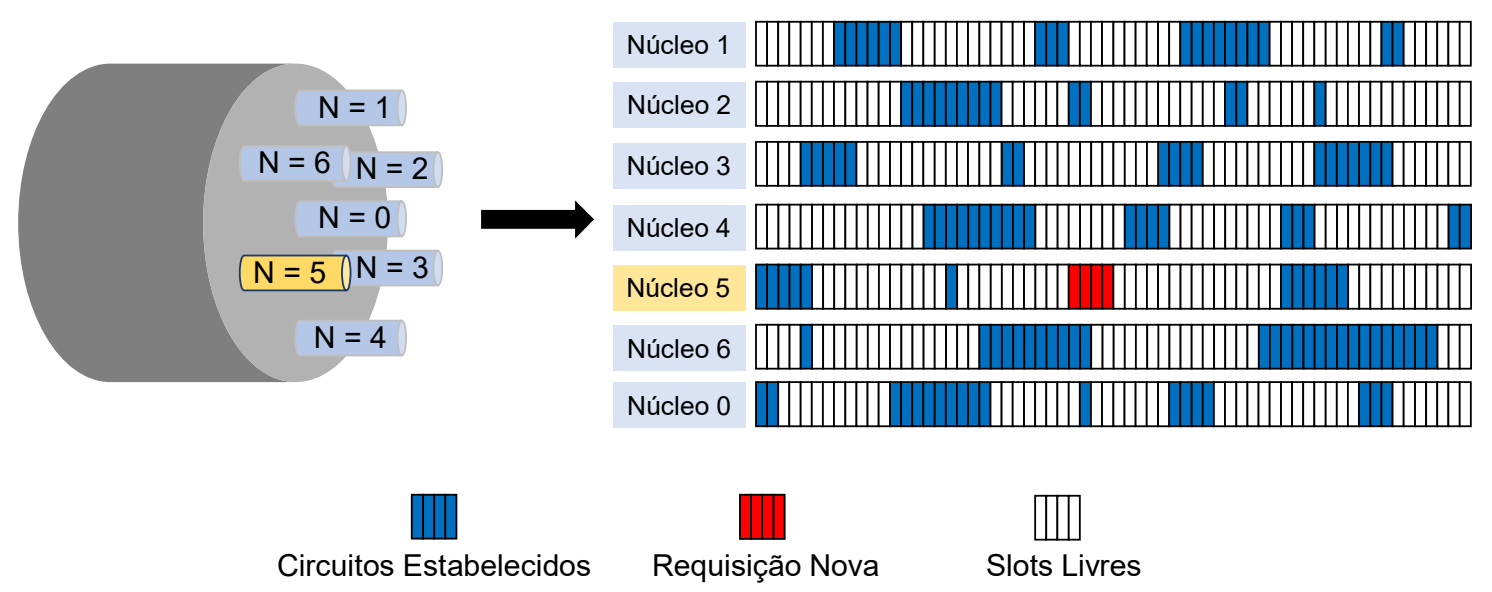

Figura 2. Escolha de núcleo e espectro em redes EON-SDM. 
Em uma rede EON-SDM, uma solução RMCSA deve ser escolhida para o estabelecimento de um circuito óptico em atendimento a cada requisição de conexão que chega na rede. A requisição de um circuito óptico é composta pelos pares de nós origem e destino, e sua demanda por largura de banda. Após a chegada de uma requisição, o algoritmo deve escolher uma rota entre os pares de nós e deve decidir o formato de modulação para envio dos bits de sinais. A quantidade de slots necessários para atender a requisição é definida utilizando os dados de largura de banda requisitada e o formato de modulação escolhido. Com a quantidade de slots da requisição calculada, o algoritmo CSA escolhe um núcleo e um conjunto de slots que supram a demanda, como exemplificado na Figura 2. Se a rede apresentar recurso suficiente para a requisição, um circuito óptico dedicado é estabelecido. Caso contrário, o circuito óptico não é estabelecido e ocorre um bloqueio de requisição.

Durante a decisão do conjunto de slots que será usado no estabelecimento do circuito óptico, o algoritmo de escolha de espectro deve respeitar duas restrições [Chatterjee et al. 2015]: i) Contiguidade Espectral, exige que os slots escolhidos para atender uma requisição sejam adjacentes entre si no espectro óptico; ii) Continuidade Espectral, exige que o conjunto de slots escolhido para atender a requisição seja o mesmo em cada um dos enlaces ao longo da rota selecionada.

Diferentes algoritmos de alocação de slots podem ser usados para o estabelecimento do circuito, como por exemplo, os algoritmos first fit, last fit, medium fit, best fit e random fit [Chatterjee et al. 2015, Lacerda Jr. et al. 2020]. O algoritmo first fit escolhe o conjunto de slots de menor índice, ou seja, mais próximo do início do espectro. O last fit escolhe o conjunto de slots mais próximo do final do espectro, os slots de maior índice. $\mathrm{O}$ algoritmo medium fit aloca a faixa de slots mais próxima do meio do espectro óptico. O best fit, quando possível, aloca o conjunto de slots que tenha a mesma largura de banda da requisição (independente da frequência do conjunto de slots). Por fim, o random fit escolhe um conjunto de slots aleatório.

\subsection{Efeitos de degradação de sinal da camada física}

No contexto das redes ópticas elásticas, a qualidade do sinal óptico emitido sofre degradações durante seu percurso na fibra. Eventos de degradação, que surgem nos enlaces ou em outros componentes da rede, são chamados efeitos de camada física. Em uma rede EON, os níveis de qualidade de transmissão (Quality of Transmission - QoT) dos circuitos ópticos são mensurados através da relação sinal ruído óptico (Optical Signal to Noise Ratio - OSNR). A OSNR é uma relação entre a densidade espectral de potência (Power Spectral Density - PSD) do sinal do circuito com a PSD dos ruídos que o atingem. A fórmula para calculo da OSNR adotada neste trabalho é apresentada em [Johannisson and Agrell 2014, Yan et al. 2015].

Além dos efeitos de camada físicas presentes nas redes EON, a adição da multiplexação por divisão espacial traz o fenômeno do crosstalk entre núcleos (XT). O crosstalk ocorre quando dois núcleos adjacentes utilizam a mesma frequência (slot) para o estabelecimento de circuitos ópticos. Quanto menor a distância espacial entre dois núcleos, maior a incidência de crosstalk sobre os circuitos [Lacerda Jr. et al. 2020]. Em [Lobato et al. 2019] é proposto um modo de mensurar a interferência do crosstalk em redes EON-SDM. A Equação 1 calcula o nível de crosstalk em um circuito óptico. 


$$
X T_{\mu, i}^{(t o t)}=\sum_{l=1}^{N_{i}} X T_{\mu, i}^{(l)}
$$

Sendo $N_{i}$ a quantidade de enlaces no circuito e $X T_{\mu, i}^{(l)}$ é o crosstalk de um enlace, que é calculado pela Equação 2.

$$
X T_{\mu, i}=\frac{P_{X T_{i}}}{P_{S_{i}}}
$$

Na Equação 2, $P_{X T_{i}}$ é o valor do crosstalk no enlace $i$ (Equação 3) e $P_{S_{i}}$ é a potencia do sinal de $i$.

$$
P_{X T_{i}}=\sum_{j=1}^{N_{a}}\left(I_{S O_{i j}} \cdot P_{S_{j}} \cdot h \cdot L\right)
$$

$N_{a}$ é o número de núcleos adjacentes a $i$. O índice $I_{S O_{i j}}$, que é calculado pela Equação 4, quantifica a sobreposição de slots entre os circuitos $i$ e $j$. A razão $h$ é calculada pela Equação 5 e $L$ é o tamanho do enlace de $i$.

$$
I_{S O_{i j}}=\frac{N_{S O_{i j}}}{N_{S_{j}}}
$$

A Equação 4 calcula a sobreposição de slots, entre os circuitos $i$ e $j$. Sendo $S O_{i j}$ a quantidade de slots alocados em $i$ que possuem mesma frequência alocada no circuito $j$ e $N_{S_{j}}$ a quantidade de slots de $j$.

$$
h=\frac{2 \cdot k^{2} \cdot R}{\beta \cdot \Lambda}
$$

Na Equação 5, $k$ é o coeficiente de acoplamento, $R$ é o raio de curvatura, $\beta$ é a constante de propagação e $\Lambda$ a distância entre os núcleos.

A Figura 3 apresenta o fluxo do processamento de requisições para alocação de circuitos considerada neste trabalho. Após a requisição de um novo circuito óptico, o algoritmo RMCSA escolhe a rota, o nível de modulação, o núcleo e o conjunto de slots de frequência. Caso não existam recursos disponíveis para alocação, a requisição é bloqueada. Existindo recursos disponíveis na rota para atender a banda requisitada, é analisado a QoT dos circuitos através da OSNR. Caso a QoT no circuito candidato não esteja adequada, a requisição é bloqueada por ausência de QoT no novo circuito (QoTN). Caso a QoTN esteja dentro dos limiares de OSNR, é analisado o impacto da alocação do novo circuito na QoT dos circuitos já estabelecidos. Se a alocação do novo circuito causar interferência significativa na OSNR dos circuitos já estabelecidos, tornando-os os seus OSNR inaceitáveis, ocorre um bloqueio de circuito por ausência de QoT nos outros circuitos (QoTO). Por fim, se os valores de QoTN e QoTO forem adequados, o crosstalk inter núcleos é avaliado, caso o valor esteja dentro limiar, o circuito é estabelecido. Senão o circuito é bloqueado por crosstalk. 


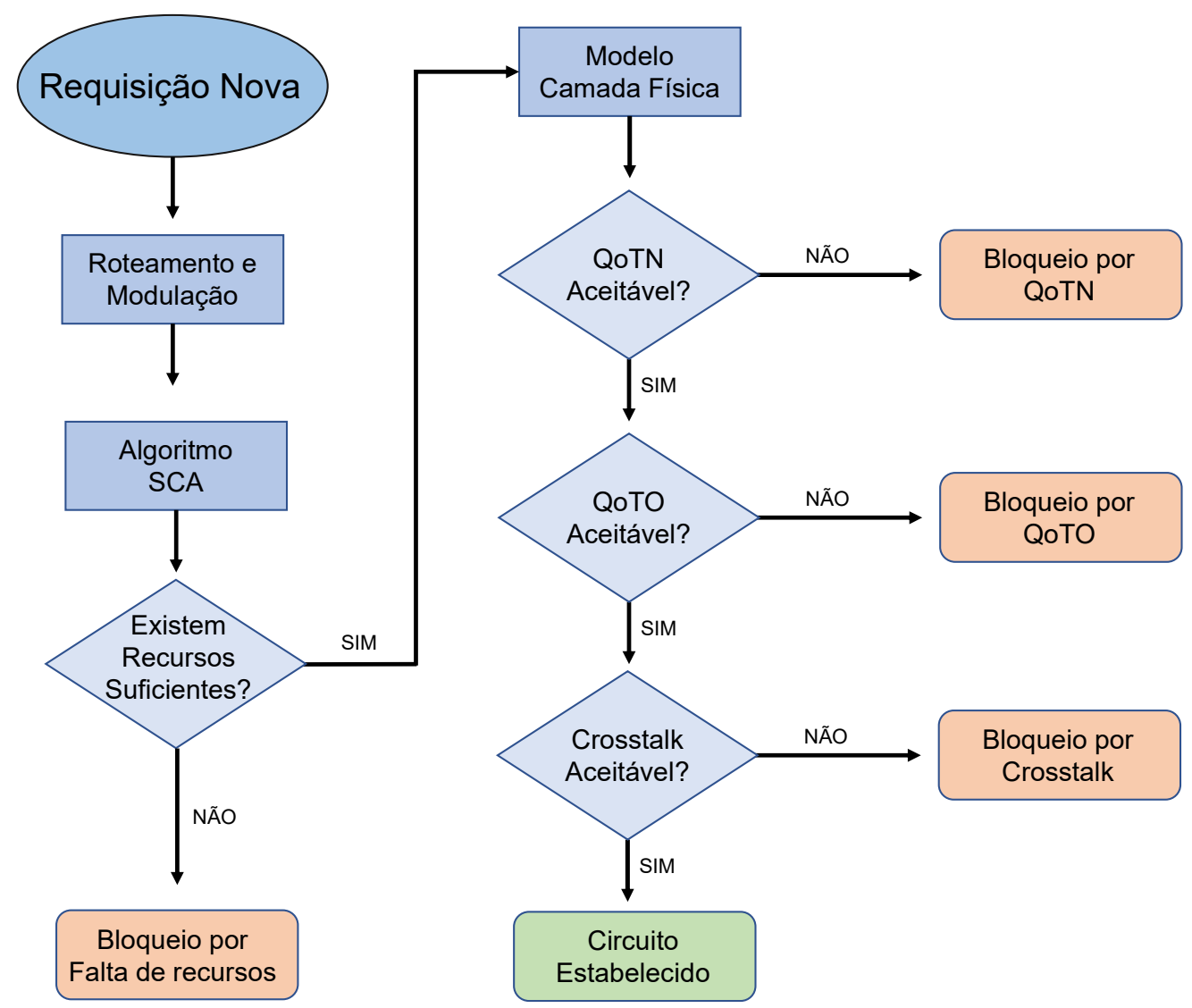

Figura 3. Fluxograma do processo de estabelecimento de um circuito óptico.

\section{Trabalhos Relacionados}

As redes EON-SDM têm ganhado cada vez mais destaque como alvo de estudo na comunidade científica. A interferência do crosstalk nos circuitos e o subproblema de escolha de núcleo e espectro são algumas das questões frequentemente abordadas na literatura. Além dos novos problemas adicionados, também são abordados outros efeitos de camada física e variações do problema de roteamento e modulação, agora no contexto das redes SDM.

Em [Fujii et al. 2014], os autores buscam diminuir os níveis de crosstalk e fragmentação na rede. O trabalho propõe um método dinâmico de priorização de núcleos baseado na estrutura da fibra multi-núcleo. Os autores também apresentam um sistema de classificação de núcleos baseado nas larguras de bandas requisitadas para os circuitos.

Os autores em [Moura and Fonseca 2018] apresentam um algoritmo RCMLSA (Routing, Core, Modulation Level, and Spectrum Assignment) de baixa complexidade computacional para redes EON-SDM. Os autores propõe uma representação matricial do espectro óptico e a utilização de algoritmos de reconhecimento de imagens para encontrar um conjunto de slots livres.

Os autores em [Savva et al. 2018] apresentam um método escalonável de categorização de núcleos para MCF’s. Também é proposto dois modelos de divisão do espectro em grupos. Além de propor métodos de alocação de núcleo e espectro, os autores 
propõe um modelo de reprocessamento de requisições bloqueadas denominado Feedbackbased Process: QoT-aware Approach.

Os autores em [Oliveira and Fonseca 2019] propuseram um algoritmo de proteção para redes EON-SDM baseado em roteamento multicaminho e caminhos de backup compartilhados. O algoritmo busca diminuir a fragmentação gerada pela alocação e desalocação de circuitos. Além da diminuição do bloqueio por fragmentação, o algoritmo traz um incremento de sobrevivência para a rede.

Em [Lobato et al. 2019], os autores propuseram um algoritmo RSCA para redes EON-SDM que leva em consideração o crosstalk na rede. O algoritmo proposto busca minimizar a incidência de crosstalk no novo circuito e nos circuitos já estabelecidos. $\mathrm{O}$ algoritmo cria um conjunto de soluções para a requisição e escolhe o circuito candidato com o valor de crosstalk mais distante do limiar estabelecido.

Em [Lacerda Jr. et al. 2020] é proposto um algoritmo de alocação de núcleo e espectro para redes EON-SDM. O algoritmo proposto busca diminuir a ocorrência de crosstalk nos circuitos dividindo os núcleos em grupos e utilizando uma política de alocação diferente para cada grupo.

Apesar de vários trabalhos na literatura considerarem que a escolha do núcleo pode interferir nos níveis de crosstalk da rede, não foram encontrado métodos que buscam utilizar os núcleos de forma a beneficiar o núcleo mais distante espacialmente dos núcleos anteriormente utilizados. Este trabalho apresenta um novo mecanismo de escolha de núcleo baseado em uma tabela de pontuação. O mecanismo tem como objetivo aumentar a prioridade do núcleo visando reduzir a incidência de crosstalk.

A busca por uma maneira de utilizar os recursos ópticos de forma eficiente e por um método de diminuir os impactos causado pelo crosstalk é frequentemente explorada na literatura. Os trabalhos que consideram o crosstalk na hora de alocar um circuito, de maneira geral, podem ser classificados de três maneiras [Lobato et al. 2019]: i) XT-avoid, técnica que busca evitar a sobreposição de slots em núcleos adjacentes; ii) XT-worst case, o algoritmo considera sempre o pior cenário de crosstalk; iii) XT-aware, o algoritmo toma decisões com base no valor do crosstalk atual da real. Afim de manter um baixo custo computacional, o algoritmo proposto utiliza a abordagem XT-avoid para diminuir a ocorrência de crosstalk e fazer um uso eficiente dos recursos da rede.

Alguns trabalhos da literatura dividem o espectro óptico em grupos e utilizam a abordagem XT-avoid. Apesar disso, não foi observado na literatura trabalhos que propõe políticas diferentes de alocação dentro desses grupos de espectro. Afim de suprir essa carência da literatura, é proposto um novo modelo de alocação chamado distanciamento espectral de circuitos. Esse modelo organiza as políticas de alocação dentro do espectro óptico para que o estabelecimento de um circuito óptico seja feito preferencialmente em slots diferentes para núcleos adjacentes.

\section{Algoritmo Proposto}

Este trabalho apresenta o Algoritmo de Distanciamento Espectral Inter-Núcleos (ADEIN) para redes EON-SDM. O ADEIN visa diminuir a ocorrência de crosstalk evitando alocar conjuntos de slots iguais em núcleos adjacentes e buscando utilizar o núcleo com menor potencial de causar interferências nos núcleos usados anteriormente. $\mathrm{O}$ algoritmo pro- 
posto pode ser dividido em duas partes: $i$ ) A seleção de núcleo, que ocorre utilizando um sistema pontuação que busca favorecer o núcleo com menor possibilidade de ocorrência de crosstalk; ii) A seleção de espectro, que realiza a tentativa de alocação do circuito em porções de slots com frequências diferentes para cada núcleo. A Figura 4 apresenta como é feita a divisão dos núcleos de acordo com o núcleo selecionado, a tabelas de pontuação inicial e as tabelas de pontuação a serem aplicadas.

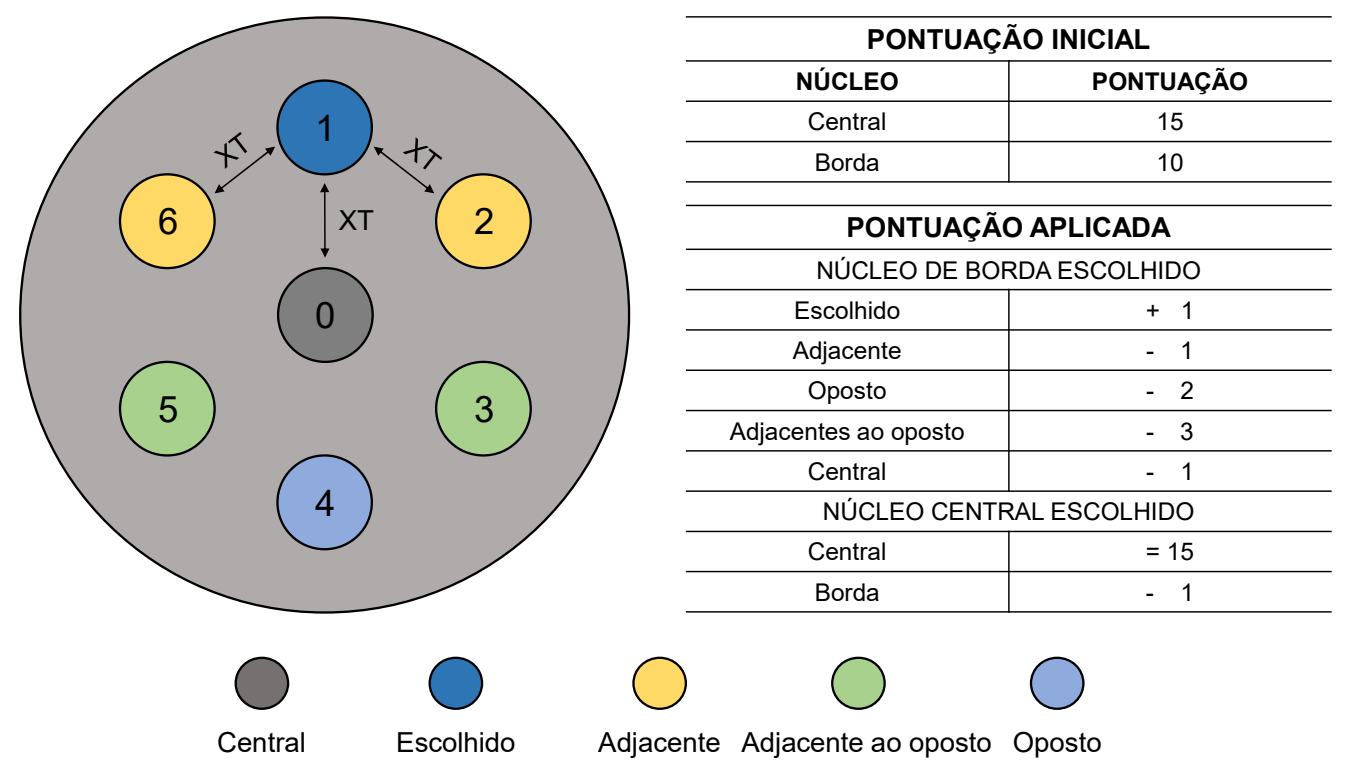

Figura 4. Categorização dos núcleos e tabelas de pontuação.

Com o intuito de selecionar um núcleo não adjacente ao núcleo anteriormente usado, a tabela de pontuação foi desenvolvida. A tabela de pontuação aplicada visa fazer com que os núcleos espacialmente mais longe do núcleo escolhido ganhem prioridade para as futuras alocações. Assim que a rede é iniciada, todos os núcleos são definidos com suas respectivas pontuações iniciais. Com a chegada de uma requisição, o algoritmo irá verificar a pontuação de cada núcleo e escolherá para alocação o núcleo com menor pontuação. Em caso de empate, é escolhido o núcleo com maior índice. Após selecionar um núcleo para a requisição do circuito, quaisquer núcleos com pontuação menor ou igual a zero terão seus pontos reiniciados. Por fim, a tabela de pontos é aplicada. Se o núcleo escolhido foi o núcleo central, os núcleos de borda perdem 1 ponto e o núcleo central é reiniciado. Caso contrário, a pontuação aplicada segue a tabela para núcleos de borda.

Após a escolha do núcleo que será usado na requisição, o algoritmo de decisão de espectro é iniciado. Como mostra a Figura 5, é atribuída uma cor a cada par de núcleo fisicamente oposto e o núcleo central compõe isoladamente uma cor. O espectro óptico é dividido em quatro porções, que serão usadas distintamente em cada cor. As três primeiras porções são para os grupos de núcleos de borda e a última porção para o núcleo central. Assim como é abordado em [Savva et al. 2018], a divisão é feita de forma que a quantidade de slots que pertence a cada porção seja proporcional a quantidade de núcleos que a compõe. Assim, para divisão do espectro são consideradas três marcações: $i$ ) T1, demarcado no slot de posição $2 / 7$ do espectro óptico; ii) T2, demarcado em 4/7 do espectro óptico; iii) T3, localizado no slot equivalente a $6 / 7$ do espectro óptico. 


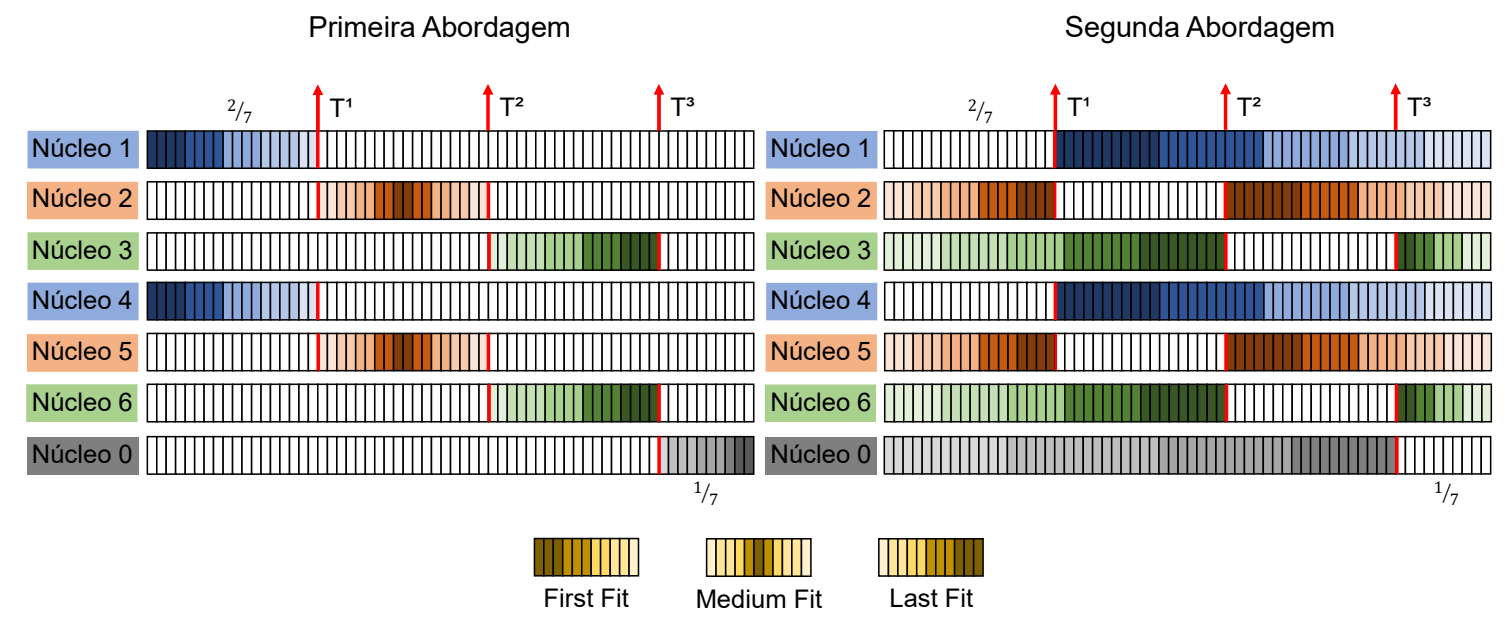

Figura 5. Modelo de divisão do espectro e sua política de alocação.

Para escolher um conjunto de slots que atenda à requisição de circuito, o algoritmo inicialmente tentará alocar utilizando a primeira abordagem e em caso de falha tentará alocar com a segunda abordagem. A Figura 5 representa as diferentes abordagens e suas políticas de alocação de circuito. Como pode-se observar, os slots são coloridos de acordo com seu grupo e sua tonalidade é definida de acordo com sua política de alocação. As alocações são feitas seguindo na ordem do tom mais forte para o tom mais fraco.

Afim de distanciar os circuitos estabelecidos em grupos diferentes, foi desenvolvida a primeira abordagem. Nela, a alocação é feita utilizando locais reservados do espectro e e obedecendo sentidos diferentes na alocação. Na primeira abordagem, o grupo azul, que contem os núcleos 1 e 4, alocará circuitos apenas nos slots com frequência menor que a marcação T1, e seguirá a política de alocação first fit. O grupo laranja, que contem os núcleos 2 e 5, faz uma alocação medium fit apenas em porções do espectro com frequência maior ou igual a T1 e menor que a marcação T2. O grupo verde, que contem os núcleos 3 e 6, irá alocar circuitos apenas em slots com frequência superior ou igual a T2 e inferior a T3, seguindo a política de alocação last fit. Por fim, o grupo preto, pertencente ao núcleo central, realizará alocação de circuitos apenas nos slots com frequência superior ou igual a T3 e seguindo a política last fit.

Com o estabelecimento e remoção de circuitos, os locais reservados para os grupos de núcleos irão fragmentar ou tornar-se totalmente preenchidos. Para isso foi desenvolvido a segunda abordagem, que aloca circuitos na porção do espectro não utilizada na primeira abordagem e prioriza alocar os slots de frequência menos utilizados na primeira abordagem. A segunda abordagem é iniciada caso a primeira abordagem não obtenha sucesso em encontrar slots livres e compatíveis. O grupo azul, irá alocar, seguindo a política first fit, slots com frequência superior a T1. O grupo laranja irá alocar, nos slots com frequência menor que T1, seguindo a política last fit e nos slots acima ou iguais de T2 seguirá a política first fit. O grupo verde alocará nos slots com frequência inferior a T2 seguindo a política last fit e nos slots superior ou igual a T3 seguindo a política first fit. Por fim, o grupo do núcleo central irá alocar apenas porções do espectro com frequência menor que $\mathrm{T} 3$. 


\section{Avaliação de Desempenho}

Para mensurar o desempenho do algoritmo proposto foi utilizado o simulador SLICE Network Simulator (SNetS) [Fontinele et al. 2017]. Em cada simulação, foram geradas 100.000 requisições, distribuidas de forma uniforme entre os pares de nós da rede. A geração das requisições segue uma distribuição de Poisson com taxa média de $\lambda$ e o tempo médio de uso dos circuitos é distribuído exponencialmente com média $1 / \mu$. Foram utilizadas as larguras de bandas 100, 150, 200, 250, 300, 350 e 400 Gbps, com proporção inversamente proporcional à largura da banda. Foram realizadas 10 replicações com diferentes sementes para geração de variáveis aleatórias. O nível de confiança adotado é de 95\%. Foram utilizadas duas topologias: $i$ ) EON, é uma topologia de alta conectividade composta por 28 nós e 88 enlaces; $i$ i) NSFNet, uma topologia de baixa conectividade composta por 14 nós e 18 enlaces [Lacerda Jr. et al. 2020].

Para o teste de desempenho, foi considerada uma MCF de 7 núcleos, com cada núcleo contendo 320 slots de frequência com largura de banda de 12,5 GHz. Foi utilizado um slot como banda de guarda. Outros parâmetros de camada física utilizados na simulação são apresentados na Tabela 1 [Lobato et al. 2019]

Tabela 1. Parâmetros utilizadas na camada física.

\begin{tabular}{c|c}
\hline Descrição & Valor \\
\hline Densidade espectral de potência do sinal & $-23 \mathrm{dBm} / \mathrm{GHz}$ \\
Atenuação da fibra $(\alpha)$ & $0,2 \mathrm{~dB} / \mathrm{km}$ \\
Parâmetro de dispersão da fibra $(D)$ & $16 \mathrm{ps} / \mathrm{nm} / \mathrm{km}$ \\
Coeficiente não-linear da fibra $(\gamma)$ & $1,3(\mathrm{Wkm})^{-1}$ \\
Tamanho de um span $\left(L_{s}\right)$ & $80 \mathrm{~km}$ \\
Figura de ruído do amplificador $(N F)$ & $5 \mathrm{~dB}$ \\
Raio de curvatura $(R)$ & $0,01 \mathrm{~m}$ \\
Constante de propagação $(\beta)$ & $10^{7} \mathrm{~m}^{-1}$ \\
Distância entre núcleos $(\Lambda)$ & $4,5 \times 10^{-5} \mathrm{~m}$ \\
Coeficiente de acoplamento $(k)$ & $5,84 \times 10^{-3} \mathrm{~m}^{-1}$ \\
\hline
\end{tabular}

Para escolha da rota, o algoritmo de Dijkstra é utilizado. Após escolha do caminho que será utilizado no circuito óptico, o formato de modulação pode ser calculado. A escolha do formato de modulação leva em consideração a distância do caminho escolhido e a distância máxima assegurada pela modulação. Os formatos de modulação utilizados são BPSK, QPSK, 8QAM, 16QAM, 32QAM. Na Tabela 2 é apresentada a distância máxima suportada, o limiar de OSNR por símbolo e o limiar de XT [Gong et al. 2013, Ives et al. 2015, Ehsani Moghaddam et al. 2019].

Tabela 2. Parâmetros utilizados para modulação

\begin{tabular}{c|c|c|c|c|c}
\hline & BPSK & QPSK & 8QAM & 16QAM & 32QAM \\
\hline Distância & $10.000 \mathrm{~km}$ & $5.000 \mathrm{~km}$ & $2.500 \mathrm{~km}$ & $1.250 \mathrm{~km}$ & $650 \mathrm{~km}$ \\
Limiar de OSNR & $5,5 \mathrm{~dB}$ & $8,5 \mathrm{~dB}$ & $12,5 \mathrm{~dB}$ & $15,1 \mathrm{~dB}$ & $18,1 \mathrm{~dB}$ \\
Limiar de XT & $-14 \mathrm{~dB}$ & $-18,5 \mathrm{~dB}$ & $-21 \mathrm{~dB}$ & $-25 \mathrm{~dB}$ & $-27 \mathrm{~dB}$ \\
\hline
\end{tabular}

O algoritmo proposto foi comparado com outros três algoritmos SCA XT-Avoid: i) Algoritmo de Balanceamento Inter-Núcleo (ABNE) [Lacerda Jr. et al. 2020]; ii) Core 
Priorization with First Fit (CP-FF) [Fujii et al. 2014] e iii) Core Priorization with Random Fit (CP-RF) [Fujii et al. 2014]. As métricas utilizadas foram probabilidade de bloqueio de banda (PBB), probabilidade de bloqueio de circuito $(\mathrm{PBC})$ e probabilidade de bloqueio de banda por crosstalk (PBB XT).

a) PBB EON

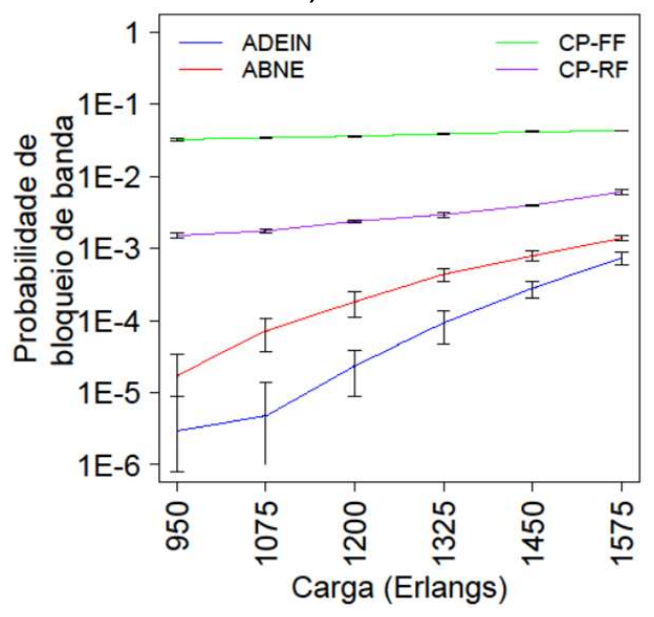

c) PBC EON

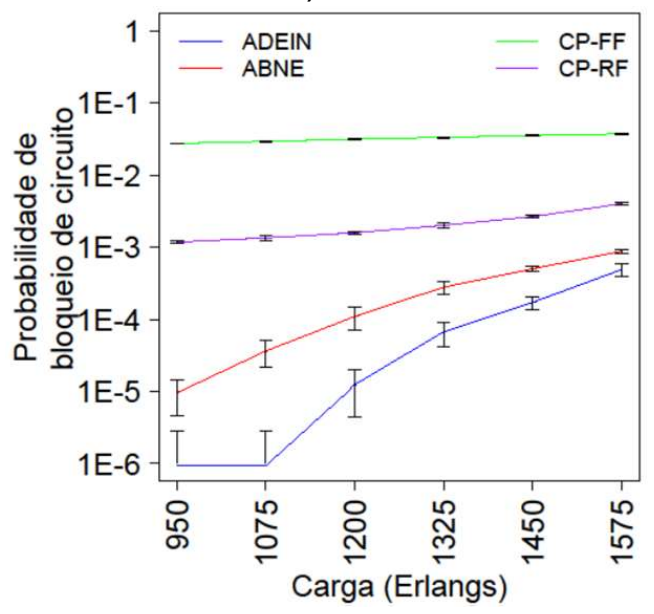

b) PBB NSFNET

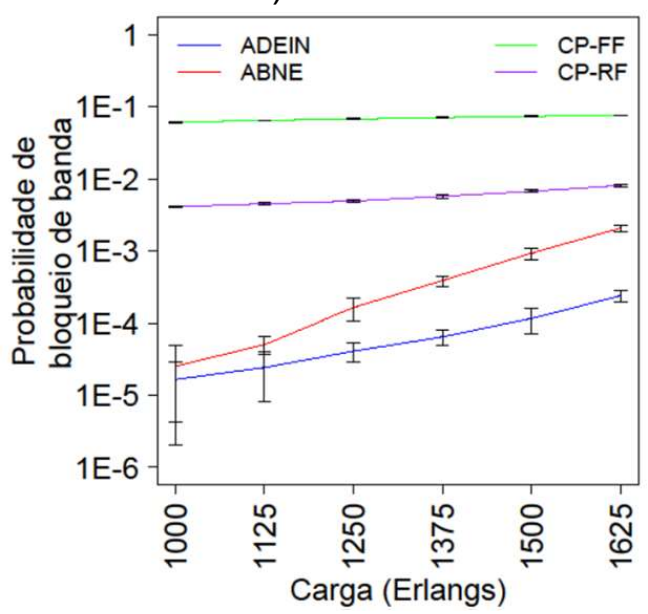

d) PBC NSFNET

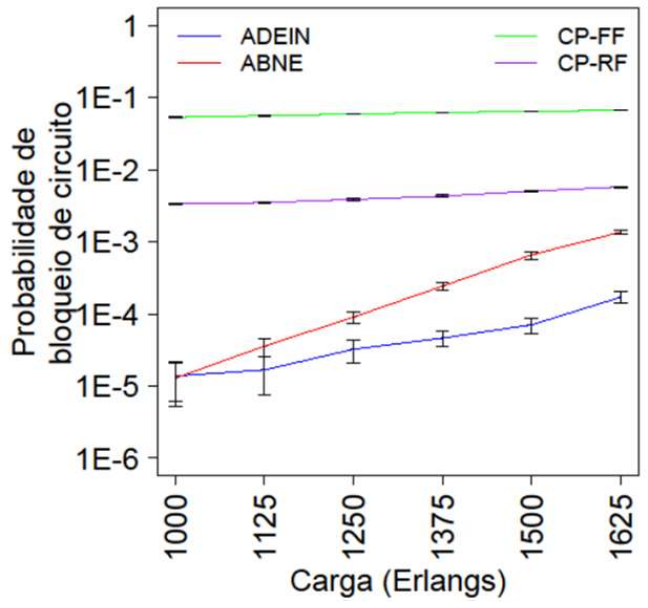

Figura 6. Gráficos de Probabilidade de Bloqueio.

A Figura 6 mostra o desempenho dos algoritmos nas métricas de probabilidade de bloqueio de banda e probabilidade de bloqueio de circuito. Nas duas métricas o comportamento é semelhante, então usaremos como foco apenas a PBB. Como evidencia a Figura 6 a) e 6 b), o algoritmo ADEIN obteve os melhores resultados de probabilidade de bloqueio de banda em comparação aos concorrentes avaliados. Para pontos de carga maiores, os algoritmos ADEIN e ABNE tendem a ter comportamento semelhantes, isso porque para cargas mais altas o bloqueio por falta de recurso começa a ter mais impacto do que o bloqueio por crosstalk. Os algoritmos CP-FF e CP-RF apresentaram resultados de probabilidade de bloqueio sem muita variação com o aumento da carga, e em níveis de bloqueio superiores ao ADEIN e ao ABNE. Entre os pontos de carga sem sobreposição dos intervalos de confiança, o algoritmo ADEIN em comparação com o ABNE obteve ganhos na probabilidade de bloqueio de banda de pelo menos $47,01 \%$ na topologia EON 
e de 74,97\% na topologia NSFNet. Em comparação com os algoritmos CP-FF e CP-RF, para topologia EON, o ADEIN obteve uma melhora mínima de respectivamente 98,29\% e 87,97\%. Ainda em relação aos algoritmos CP-FF e CP-RF, na topologia NSFNet, o ADEIN obteve ganhos de PBB em pelo menos 99,68\% e 97,03\%, respectivamente.
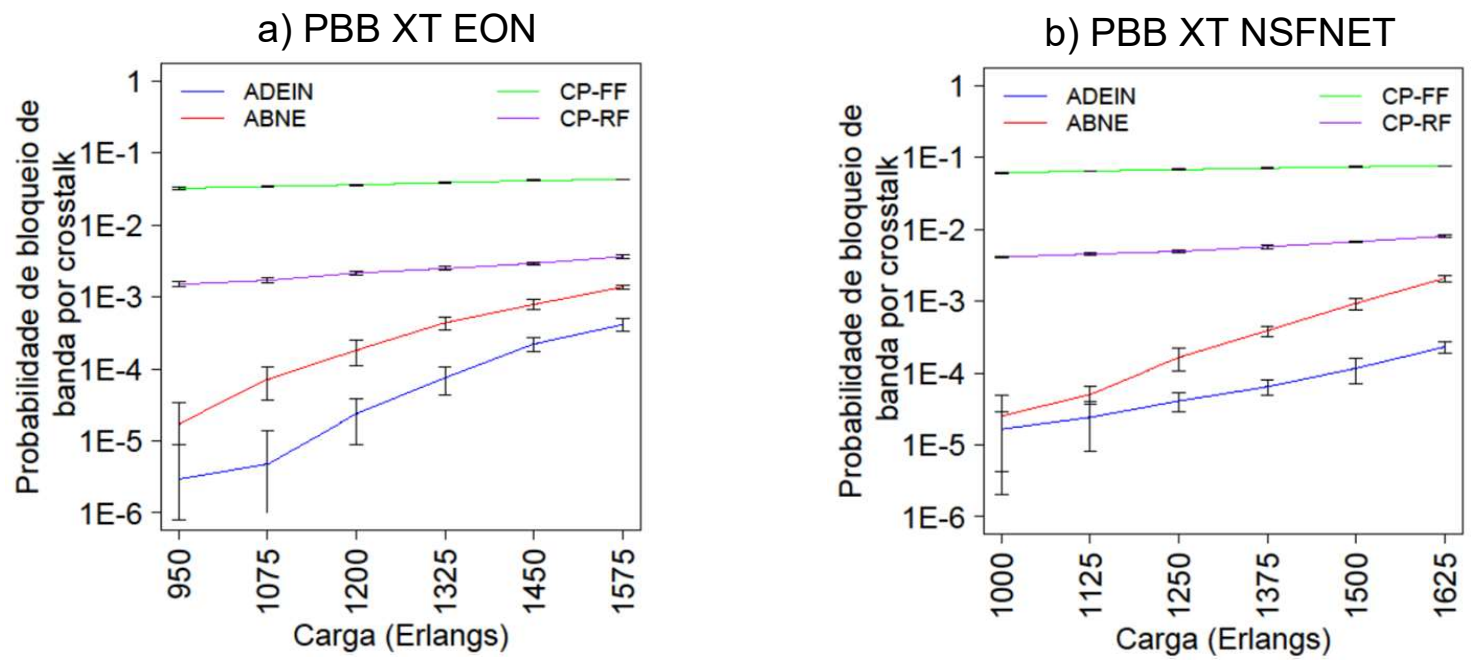

Figura 7. Probabilidade de bloqueio por crosstalk.

A Figura 7 apresenta a probabilidade de bloqueio de banda por crosstalk. Nas cargas mais baixas, os algoritmos ADEIN e ABNE apresentam comportamento semelhante, devido aos seus primeiros bloqueios motivados pela natureza do núcleo central. Como aumento da carga de tráfego, o ADEIN apresentou resultados melhores do que todos os outros algoritmos avaliados, para ambas as topologias. Em pontos sem sobreposição do intervalo de confiança, o algoritmo proposto apresentou uma melhora em relação ao ABNE de pelo menos $69,98 \%$ na topologia EON e 74,97\% na topologia NSFNet. Em comparação com os algoritmos CP-FF e CP-RF, na topologia EON, o ADEIN obteve uma melhora de no mínimo $99,04 \%$ e $88,73 \%$, respectivamente. Para topologia NSFNet, em comparação com o CP-FF e CP-RF o ADEIN obteve melhora mínima de respectivamente $99,69 \%$ e $97,11 \%$.

O algoritmo proposto apresentou uma melhor utilização dos recursos ópticos em relação aos outros algoritmos avaliados. Apesar do ABNE também propor uma forma de balancear o espectro, o modelo de divisão do espectro em grupos de núcleos e o distanciamento espectral da proposta demostrou melhores resultados. A utilização de uma técnica de seleção balanceada dos núcleos combinada com a distribuição dos circuitos de forma distanciada no espectro faz com que a proposta se sobressaia, diminuindo os níveis de crosstalk na rede. O melhor desempenho do algoritmo proposto em reduzir os níveis do crosstalk evidencia que modelos de seleção de núcleo e métodos de alocação de espectro são mais efetivos quando utilizados em conjunto.

\section{Conclusão}

Neste trabalho foi proposto o Algoritmo de Distanciamento Espectral Inter-Núcleos (ADEIN). A proposta busca reduzir a ocorrência de crosstalk na rede utilizando duas estratégias: $i$ ) Escolha de núcleo baseado em classificação e pontuação; $i i$ ) Escolha de 
espectro visando distanciar ao máximo os locais de alocação em núcleos adjacentes. O algoritmo proposto foi comparado com os algoritmos ABNE, CP-FF e CP-RF. Foram utilizadas as métricas de probabilidade de bloqueio de banda por crosstalk, probabilidade de bloqueio de banda e probabilidade de bloqueio de circuito.

Ao aplicar as estratégias do algoritmo ADEIN observou-se a redução da incidência de crosstalk e consequentemente um menor bloqueio de requisições. Na topologia EON, o algoritmo proposto chegou a obter uma melhora na probabilidade de bloqueio de banda de pelo menos 47,01\% em relação ao ABNE e de no mínimo 98,29\% e 87,97\% em relação ao CP-FF e CP-RF, respectivamente. Para topologia NSFNet, o ADEIN obteve melhora mínima de 74,97\% em relação ao ABNE e de 99,68\% e 97,03\% em relação ao CP-FF e CP-RF, respectivamente. Ainda que técnicas de alocação e técnicas de seleção de núcleo apresentem ganhos quando utilizadas isoladamente, utilizar essas técnicas em conjunto e distanciar a alocação inter-núcleo trará melhores resultados na mitigação do crosstalk.

Em trabalhos futuros, pretende-se desenvolver novas versões para o ADEIN, expandir o cenário de avaliação, utilizar novas métricas e comparar com outras propostas da literatura. Planeja-se também, evoluir o algoritmo de seleção de núcleo e desenvolver novas tabelas de pontuação afim de iniciar uma nova abordagem de seleção.

\section{Referências}

Chatterjee, B., Sarma, N., and Oki, E. (2015). Routing and spectrum allocation in elastic optical networks: a tutorial. IEEE Communications Surveys Tutorials, 17(3):1776 1800 .

Ehsani Moghaddam, E., Beyranvand, H., and Salehi, J. A. (2019). Crosstalk-aware resource allocation in survivable space-division-multiplexed elastic optical networks supporting hybrid dedicated and shared path protection. Journal of Lightwave Technology, pages 1-1.

Fontinele, A., Santos, I., Neto, J. N., Campelo, D. R., and Soares, A. (2017). An efficient IA-RMLSA algorithm for transparent elastic optical networks. Computer Networks, 118:1 - 14 .

Fujii, S., Hirota, Y., Tode, H., and Murakami, K. (2014). On-demand spectrum and core allocation for reducing crosstalk in multicore fibers in elastic optical networks. IEEE/OSA Journal of Optical Communications and Networking, 6(12):1059-1071.

Gong, L., Zhou, X., Liu, X., Zhao, W., Lu, W., and Zhu, Z. (2013). Efficient resource allocation for all-optical multicasting over spectrum-sliced elastic optical networks. IEEE/OSA Journal of Optical Communications and Networking, 5(8):836-847.

Ives, D. J., Bayvel, P., and Savory, S. J. (2015). Routing, modulation, spectrum and launch power assignment to maximize the traffic throughput of a nonlinear optical mesh network. Photonic Network Communications, 29(3):244-256.

Jinno, M., Takara, H., Kozicki, B., Tsukishima, Y., Sone, Y., and Matsuoka, S. (2009). Spectrum-efficient and scalable elastic optical path network: architecture, benefits, and enabling technologies. IEEE Communications Magazine, 47(11):66-73. 
Johannisson, P. and Agrell, E. (2014). Modeling of nonlinear signal distortion in fiberoptic networks. Journal of Lightwave Technology, 32(23):4544-4552.

Klinkowski, M. and Zalewski, G. (2019). Dynamic crosstalk-aware lightpath provisioning in spectrally-spatially flexible optical networks. IEEE/OSA Journal of Optical Communications and Networking, 11(5):213-225.

Lacerda Jr., J., Fontinele, A., Santos, I., Leão, E., Campelo, D., Monteiro, J. A., and Soares, A. (2020). Algoritmo de balanceamento inter-núcleos para redes Ópticas elásticas com multiplexação por divisão espacial. In Anais do XXXVIII Simpósio Brasileiro de Redes de Computadores e Sistemas Distribuídos, pages 519-532, Porto Alegre, RS, Brasil. SBC.

Lobato, F. R., Jacob, A., Rodrigues, J., Cartaxo, A. V. T., and Costa, J. (2019). Inter-core crosstalk aware greedy algorithm for spectrum and core assignment in space division multiplexed elastic optical networks. Optical Switching and Networking, 33:61 - 73.

Moura, P. M. and Fonseca, N. L. S. (2018). Routing, core, modulation level, and spectrum assignment based on image processing algorithms. IEEE/OSA Journal of Optical Communications and Networking, 10(12):947-958.

Oliveira, H. M. N. S. and Fonseca, N. L. S. (2018). Protection, routing, modulation, core, and spectrum allocation in SDM elastic optical networks. IEEE Communications Letters, 22(9):1806-1809.

Oliveira, H. M. N. S. and Fonseca, N. L. S. (2019). Proteção e roteamento multicaminho em redes ópticas elásticas com multiplexação por divisão espacial. Simpósio Brasileiro de Redes de Computadores e Sistemas Distribuídos SBRC 2019, pages 487-500.

Santos, I. G., Hiago, L., Fontinele, A., Monteiro, J. A. S., Soares, A., and Campelo, D. (2019). Um mecanismo para potencialização da agregação de tráfego em redes ópticas elásticas. In Anais do XXXVII Simpósio Brasileiro de Redes de Computadores e Sistemas Distribuídos, pages 321-334, Porto Alegre, RS, Brasil. SBC.

Savva, G., Ellinas, G., Shariati, B., and Tomkos, I. (2018). Physical layer-aware routing, spectrum, and core allocation in spectrally-spatially flexible optical networks with multicore fibers. In 2018 IEEE International Conference on Communications (ICC), pages $1-6$.

Trindade, S. and da Fonseca, N. L. S. (2020). Core and spectrum allocation for avoidance of spectrum fragmentation in eon-sdm. In ICC 2020 - 2020 IEEE International Conference on Communications (ICC), pages 1-6.

Yan, L., Agrell, E., Wymeersch, H., Johannisson, P., Di Taranto, R., and Brandt-Pearce, M. (2015). Link-level resource allocation for flexible-grid nonlinear fiber-optic communication systems. IEEE Photonics Technology Letters, 27(12):1250-1253. 